

\title{
On the Spatial and Temporal Accuracy of Overset Grid Methods for Moving Body Problems
}

\author{
Robert L. Meakin * \\ Overset Methods, Inc. \\ at NASA Ames Research Center M/S 258-1 \\ Moffett Field, CA 94035-1000
}

\begin{abstract}
A study of numerical attributes peculiar to an overset grid approach to unsteady aerodynamics prediction is presented. Attention is focused on the effect of spatial error associated with interpolation of intergrid boundary conditions and temporal error associated with explicit update of intergrid boundary points on overall solution accuracy. A set of numerical experiments are used to verify whether, or not, the use of simple interpolation for intergrid boundary conditions degrades the formal accuracy of a conventional second-order flow solver, and to quantify the error associated with explicit updating of intergrid boundary points. Test conditions correspond to the transonic regime. The validity of the numerical results presented here are established by comparison with existing numerical results of documented accuracy, aud by direct comparison with experimental results.
\end{abstract}

\section{INTRODUCTION}

Computation of unsteady viscons flows for grometrically complex bodies involving relative motion between component parts represents an important class of problems for which accurate methods of prediction are required. There are numerous applications of this type; lannch vehicle staging, aircraft store separation, crew escape mechanisms, and liclicopter rotor/body interaction. Present engincering tools are inadequate for risk-free analysis of this class of problems, and trial-and-error testing has become too expensive and time-consuming. Mature computational methods such as empirically-modified, threc-dimensional panel codes and nonlinear potential methods have been applied to these problems, but have not been completely successful. Unsteady viscous flowfields involving noving shocks, vortical wakes, interference effects and body motion demand the most advanced computational technicques available.

Currently, the only viable high-order method of prediction for these problems is the so called Chimera[1], or overset grid approach. The approach involves the decomposition of problen geometry into a number of geometrically simple overlapping component grids. Grid components associated with moving bodies move with the bodies without stretching or dist orting the grid sys-

* Staff Scientist, Member AIAA

Copyright (C)1994 by the Anerican Institute of Acronatics and Astronantics, Inc. All rightits rescrved.

858 tem. The structure of individual grid components facilitates viscous boundary layer resolution, and the use of implicit time-integration algorithms which are not held to the prohibitively low time-step limits characteristic of explicit schemes.

It is reasonable to presume that a solution method that has been verified to accurately predict the flow dynamics for a given application would also provide an accurate prediction for a now application, providing that the new geometry and flow conditions do not vary widely from those of the verified point of reference. If this were not the case, there would be no point in using computational methods of prediction, since construction of the test article, or prototype, would always be needed to verify the correctness of the prediction. Overset grid methods have been applied to a wide variety of problems and flow regimes. Careful verification studies have been carried ont for many non-moving body cases $[2,3,4,5,6]$. However, data sets are usually only complete enough to verify the correctness of surface pressure predictions, and verification of moving body problems is practically impossible except for ideal cases. Accordingly, verification of the predictive ability of overset grid methods is an ongoing process. Of course, this is true of methods for Computational Fluid Dynamics (CFD) in general.

The objective of the present work is to explore basic attributes peculiar to an overset grid approach as they relate to accuracy in predicting the unsteady aerodynamic fields in moving body problems. The formal solution accuracy of the basic Navier-Stokes solver employed here[7] will be taken as a given. Attention is focused on the effect of spatial crror associated with interpolation of intergrid boundary conditions and temporal error associated with explicit update of intergrid boundary points on overall solution accuracy.

\section{DISCUSSION}

An overset grid discretization of the space about geometrically complex bodies is comprised of a system of overlapping body-fitted gricls and topologically simple background grids. Tho borly-fitted grids extend a relatively short distance from body surfaces and are overset 
maintain formal solution accuracy. In practical applications, given a fixed number of grid points, it is not possible to provide grid resolution of sufficient density to guarantee that flow feat ures will always be smoothly represented in the grid. If a conservative interpolation scheme (e.g., see reference [11]) is used at intergrid boundaries, the speed and structure of flow features (i.e., shocks, vortices, etc.) can be maintained through grid interfaces. However, lacking sufficient grid resolution, the accuracy of the solution cannot be ensured in any case. Hence, the issue with domain connectivity is not necessarily one of conservative versus nonconservative interpolation, but one of grid resolution.

If solution adaption is used to ensure smooth variation of dependent variables throughout the computational space of overset grid systems, the resulting solutions will accurately approximate the governing differential equations in all respects. This point is demonstrated in subsequent paragraphs of this paper via a set of numerical experiments. The method of solution adaption suggested here is that of oversetting fine grid components where flow gradients/error indicators are high, as opposed to resorting to point redistribution methods.

\section{Steady Transonic Airfoil Examples}

Consider the stearly flow over a NACA 0012 airfoil at Mach 0.8 freestream conditions and $1.25^{\circ}$ angle-ofattack. These conditions result in a strong shock on the airfoil upper surface, and a very weak shock on the lower surface (see Figure 2). This flow situation has been used as a benchmark test condition for an AGARD advisory report on inviscid flow ficld methods[12]. In order to use the AGARD results as a point of reference for the present discussion, inviscid conditions lave been imposed here also. This simplification in no way diminishes this case as a test of the effect of intergrid interpolation on solution accuracy.

The approach here is to carry out a grid refincment study and observe the rate at which the numerical error tends to zero. The crror should decay at a second order rate since both the interior differencing scleme of the flow solver and the intergrid boundary interpolation scheme are second order. In order to correctly determine the numerical error associated with the present overset grid solutions, the exact Euler solution must be available. Cnfort unately, the exact solution is unknown. Therefore, the single very fine grid solution shown in Figure 2 is used in lien of the exact solution. The single very fine grid solution is reforred to leereafter as STAS-1 (Steady Transonic Airfoil - Single gricl - case 1).

The STA-S-1 solution was obtained using 643 points in the azimuthal direction (J), and 131 points in the surface normal direction (K). Since the flow solver used lere is fully 3D, the present 2D problem was simulated using 3 planes of $643 \times 131$ points. This spacing is twice that of the finest AGARD solution. In addition to STAS-1, four overset grid solutions of the same problem were carried out using grids of varying resolution. The naming convention adopted for the overset grid solutions is STA-M-X. The "M" stands for multiple grid case, and " $\mathrm{X}$ " stands for the particular case number referenced in Table 1. Table 1 gives statistics of the grid systems for each case presented in this section.

The overset grids are ach composed of a body-fitted grid for the airfoil, and a background Cartesian grid of corresponding resolution. The body-fitted grids are each subsets of STA-S-1. For example, STA-M-1 is identical with STA-S-1 for all points in J and all points in $K$ out to $K=61$. STA-M-2 was obtained by using every other point from STA-M-1 in both the $J$ and $K$ directions. STA-M-3 was obtained by using every third point in $\mathrm{J}$ and $\mathrm{K}$. STA-M-4 was obtained by using every sixth point in $J$ and $\mathrm{K}$. Since all overset grids have points in common with STA-S-1, the point-wise numerical error in each overset solution can be computed as a simple difference with the STA-S-1 solution. Figure 3 shows a plot matrix of the error which resulted on each overset grid solution. The top row shows solution error in the coarse overset solution (STA-M-4) with respect to the single very fine gricl solution (STA-S-1). Moving from top-to-bottom, the crror plots correspond to increasingly fine overset grid solutions. The first column of plots in Figure 3 is the local error in mass density. The sccond and third columus are the local error in $\mathrm{X}$-momentum and total energy, respectively. Figure 4 shows the rate at which the error decays as a function of grid resolution. The error represented in Figure 4 is the rms error of the crror fickls shown in Figure 3. The solid lines showı in Figure 4 represent first and second order slopes (i.e., 2nd order implies that doubling the number of grid points will recluce the error by a factor of 4).

The error reduction from the STA-M-4 to STA-M-3 solutions is second order in all flow variables. This is also true for the scalar variables $(\rho$ and $e$ ) throughout the range of grid refinement. However, the vector variables $(\rho U$ and $\rho W$ ) drop to a nearly first order slope between the STA-M-3 and STA-11-2 solutions. The reason for this is that the reference solution (STA-S-1) is not exact. As the resolution of the overset grids approaches that of the reference STA-S-1 case, the error computations become invalicl.

Surface $C_{p}^{\prime}$ plots are prescuted in Figures 5 and 6 . Figure 5 provides a comparison with the finest AGARD solution and the present STA-S-1 and STA-M-1 solutions. Clearly, the three solutions are in very good agreement. Figure 6 provides a comparison with the finest AGARD solution (again) and two relatively coarse-grid solutions, 
fully implicit, a temporal crror will result. Full aircraft applications can involve as many as 100 grid components, perhaps more. At what point does the temporal error associated with explicit intergrid boundary updates become significant? The second concern relates to numerical stability. As a domain is decomposed into more and more grid components, the lagging of intergrid boundaries makes the overall solution procedure more explicit. Indeed, in the limit as the domain is decomposed into as many grid components as there are grid points, the scheme is reduced (in essence) to Point Jacobi. At what point, then, does stability become a real issue?

\section{Unsteady Transonic Oscillating Airfoil Examples}

In order to explore the questions posed above, consider the case of an oscillating airfoil subject to transonic flow conditions. Specifically, consider a NACA 64A010 airfoil subject to Mach 0.796 freestream conditions and forced oscillation of constant amplitude. The specific case to be considered here is defined in Table 3 , and results in an attached boundary layer and weak moving upper and lower surface shocks. The motion of the shocks, and unsteady aerodynanic loads are driven by the amplitude and frequency of oscillation of the airfoil. As with the steady-state cases considered previously, the approach here is to determine numerical error relative to a very fine benchmark solution. The benchmark solution is referred to as UTOA-S-1 (Unsteady Transonic Oscillating Airfoil - Single grid case 1 ). Like the STA-S-1 case of the previous section, the UTOA-S-1 grid has 643 points in the azimuthal direction $(J)$, and 131 points in the surface normal direction (K) (see Figure 10). Relative to a dimensionless chord length of unity, the initial spacing away from the wall is $1 \times 10^{-6}$.

In order to isolate the error and stability concerns attributable to the explicit updating of intergrid boundary points, the UTOA-S-1 grid was decomposed in a special way to form the basis for the five multiple grid computational cases indicated in Table 4 (note that the "M" in Table 4 nomenclature indicates Multiple grid case). For example, the UTOA-S-1 grid was decomposed into the two overlapping grids inclicated in Figure 10 (UTOA-M-1). Both grid components in the UTOAM-1 grid are exact subsets of the UTOA-S-1 grid. Intergrid boundary points in the inner UTOA-M-1 grid are coincident with interior points of the outer UTOA-M-1 grid. Likewise, intergrid boundary points on the outer UTOA-M-1 grid are coincident with interior points of the inner UTOA-M-1 grid. As a result, there is no interpolation error associated with intergrid boundary condition updates. Further, the procedure is spatially conservative. The only possible source of error in the computations, relative to the LTOA-S-1 benchmark so- lution, is the explicit nature of the intergrid boundary updating procedure.

Adopting the same method of decomposition used to realize the 2 component UTOA-M-1 grid system, the UTOA-S-1 grid was decomposed into grid systems with 16 and 32 components (sec Figure 10). The 16 component grid system corresponds to the UTOA-M-2 case indicated in Table 4 . The 32 component grid system corresponds to the UTOA-M-3, UTOA-M-4, and UTOA-M-5 cases indicated in Table 4. Cases UTOAM-1 through UTOA-M-3 correspond to simulations using the same time-step size as that cmployed in UTOAS-1, and allow the temporal error resulting from explicit intergrid updating to be iclentified. Cases UTOA-M-3 through UTOA-M-5 correspond to simulations carried out on the 32 component grid system for time-step sizes of $\Delta t=0.0025,2 \times \Delta t$, and $4 \times \Delta t$, respectively. As a result, cases UTOA-M-3 through UTOA-M-5 may furnish some insight into the impact on stability of explicit intergrid boundary updates.

The physical problem being solved in all the UTOA cases indicated in Table 4 has been studied experimentally in the NASA Ames $11 \times 11$ foot wind-tunnel[13]. An attempt to verify the valiclity of the benchmark case by comparison with experiment has been carried out. However, due to circumstances ontlined below, the experimental data set employed dicl not facilitate a conclusive solution validation comparison. This not withstanding, the UTOA 'asc's do provide important reference information regarding the numerical questions immediately at hand.

The benchmark UTOA-S-1 unsteady solution was initiated from a nearly converged static (non-oscillating) solution abont the airfoil at mean angle-of-attack $\left(\alpha_{m}=\right.$ $\left.-0.21^{\circ}\right)$. The surface $C_{p}$, distribution resulting from a fully converged statir airfoil case is shown in Figure 11, along with the experincutal results. The agreement between the computation and experiment is very good. However, there is a sliglit discrepancy in $C_{p}$ magnitude over the first $40 \%$ chord of the foil. The reason for this is that the foil grometry used in the computation is based on the OSU definition of a NACA 64A010[14], rather than the coordinates published in reference[13]. Figure 12 illustrates the differences in the theoretical, experimental, and OSU definitions of the NACA 64A010 geometry. The definition used in the computations (OSU) corresponds to the theoretical definition up to about $50 \%$ cliord and then smoothly transitions to the experimental definition. Figure 11 also contains a full potential solution to this problem based on the theoretical NACA $64 A 010$ definition. The full potential solution was provided for comparative purposes only. The computed integral loads $\left(C_{1}\right.$ and $\left.C_{m}\right)$ are in good agreement with the corresponding experimental results (see tabu- 
crepancies between experiment and computed solutions obtained using an overset grid approach. The present results suggest that grid resolution is the primary issue. If it is not possible to provide sufficient grid resolution for a given problem, a conservative interface scheme is preferable, but accuracy will be compromised whether, or not, conservation has been maintained across grid interfaces. The present study suggests the use of overset fine grids as a practical means of insuring smooth representation of flow gradients in overset grid systems, and hence, maximizing solution accuracy.

The steady state STA-M grid refinement study carried out here indicates that formal solution accuracy is maintained in an overset grid system using tri-linear interpolation to supply intergrid boundary conditions. The results confirm that spatial error associated with interpolation of intergrid boundary data can be minimized via overset fine grid components. This appears to be true even if grid interfaces exist across shocks.

Explicit updating of intergrid boundary conditions did not have a significant adverse affect on solution accuracy for the present UTOA cases, for which the solution clonain was split into as many as 32 overlapping grid components. Solution error attributable to explicit updating of intergrid boundaries was observed to be proportional to the time-step size employed, however remained insignificant for all stable $\Delta t$. The present UTOA-M cases inherited no discernible stability penalties as a result of the explicit intergrid boundary updates. These results suggest that unsteady problems of practical importance (3D complex geometry) can be accurately simulated using an overset grid approach provided that the time-step size is sufficient to resolve the significant temporal gradients inherent to the problem.

\section{ACKNOWLEDGEMENTS:}

This work was carried out under NASA ARC grant NCC2-747. All computational results reported herein were carried ont on the NAS facility at NASA ARC. The author wishes to acknowledge the influence of the late Professor Joseph Steger on this work, as well as the author's present course of research. In addition, thanks are due to Drs. Kalpana Chawla and Christopher Atwood who have been very encouraging and have always been available for discussions on the issues presented herein. The support and direction provided by Dr. Jim McCroskey is also gratefully acknowledged.

\section{REFERENCES}

[1] Steger, J. L., Dougherty, F. C.., and Benck, J. A., "A Chimera Grid Scheme," Advances in Grid Generation, K. N. Ghia and U. Ghia, eds., ASME FED-Vol 5., June 1983.
[2] Martin, F. and Slotnick, J., "Flow Computations for the Space Shuttle in Ascent Mode Using Thin-Layer Navier-Stokes Equations," Progress in Astronautics and Aeronautics, Vol. 125: Applied Computational Aerodynamics, P. Henne, Ed., AIA A, 1990.

[3] Meakin, R., "Computations of the Unsteady Flow About a Generic Wing/Pylon/Finned-Store Configuration," AIAA Paper 92-4568-CP, pp. 564-580, August 1992.

[4] Lijewski, L. and Sulss, N., "Chimera-Eagle Store Separation," AIAA Paper 92-4569, August, 1992.

[5] Jordan, J., "Computational Investigation of Predicted Store Loads in Mutual Interference Flow Fields," AIAA Paper 92-4570-CP, pp. 581-591, August, 1992.

[6] Benek, J., Donegan, T., and Suhs, N., "Extended Chimera Grid Embedding Scheme with Application to Viscous Flows," AIAA Paper 87-1126-CP, pp. 283-291, 1987.

[7] Renze, K., Buning, P., and Rajagoplan, R., "A Comparative Study of Turbulence Models for Overset Grids," AIAA Paper 92-0437, January, 1992.

[8] Meakin, R., "A Now Motlıod For Establishing Inter-Grid Communication Among Systems of Overset Grids," AIAA Paper 91-1586-CP, pp. 662-671, June, 1991.

[9] Dietz, W., Jacocks, J., and Fox, J., "Application of Domain Decomposition to the Allalysis of Complex Aerodynamic Configurations," SIAM Conf. Domain Decomposition Metlıs., Houston, TX, March 1989.

[10] Brown, D., Chesshire, G., Henshaw, W., and Kreiss, O., "On Composite Overlapping Grids," 7th Internat. Conf. on Finite Element Methods in Flow Problems, Huntsville, AL, April 1989.

[11] Moon, Y. and Liou, M., "Conservative Treatment of Boundary Interfaces for Ovcrlaid Grids and Multilevel Grid Adaptions," AIAA Paper 89-1980-CP, pp. 480-494, 1989.

[12] Viviand, H., "Numerical Solutions of Two- Dimensional Reference Test Cases," AGARD-AR-211: Test Cases for Inviscid Flow Field Methods.

[13] Davis, S. and Malcoln,, G., "Experimental Unsteady Aerodynamics of Conventional and Supercritical Airfoils," NASA TM-81221, August, 1980.

[14] Olsen, J., "AGARD Stanclard Configurations for Aeroelastic Applications of Transonic Unsteady Acrodynamics," AFFDL-TM-78-6-FBR, Part III (Addendum), October, 1978. 
rms Error vs. $\mathbf{N}$

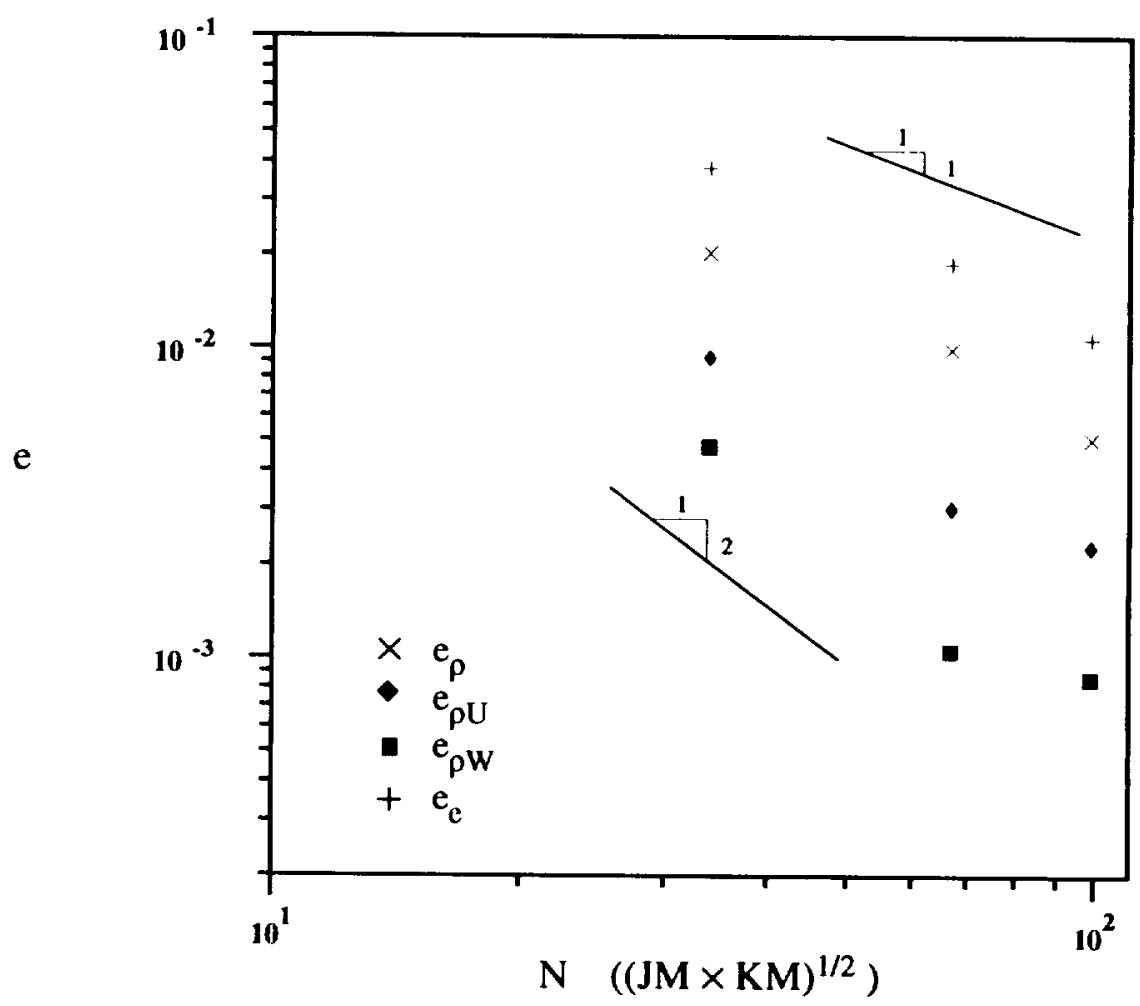

Figure 4. Grid refinement and rms error reduction for STA cases.

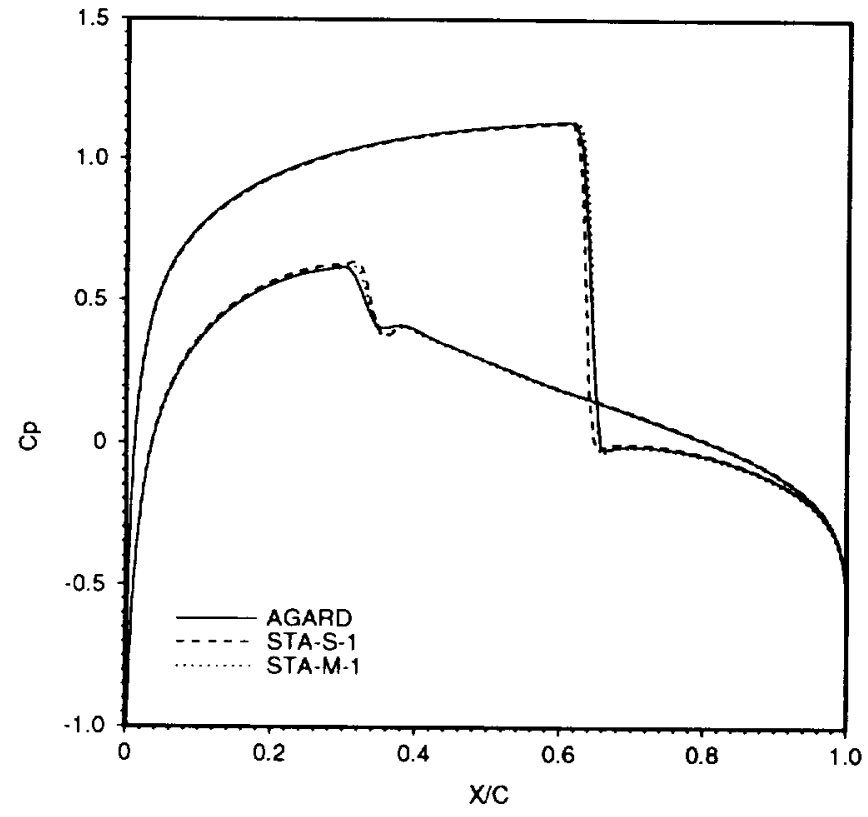

Figure 5. $C_{p}$ vs. $X / C$ plots for the finest AGARD solution [12], and the present STA-S-1 and STA-M-1 solutions.

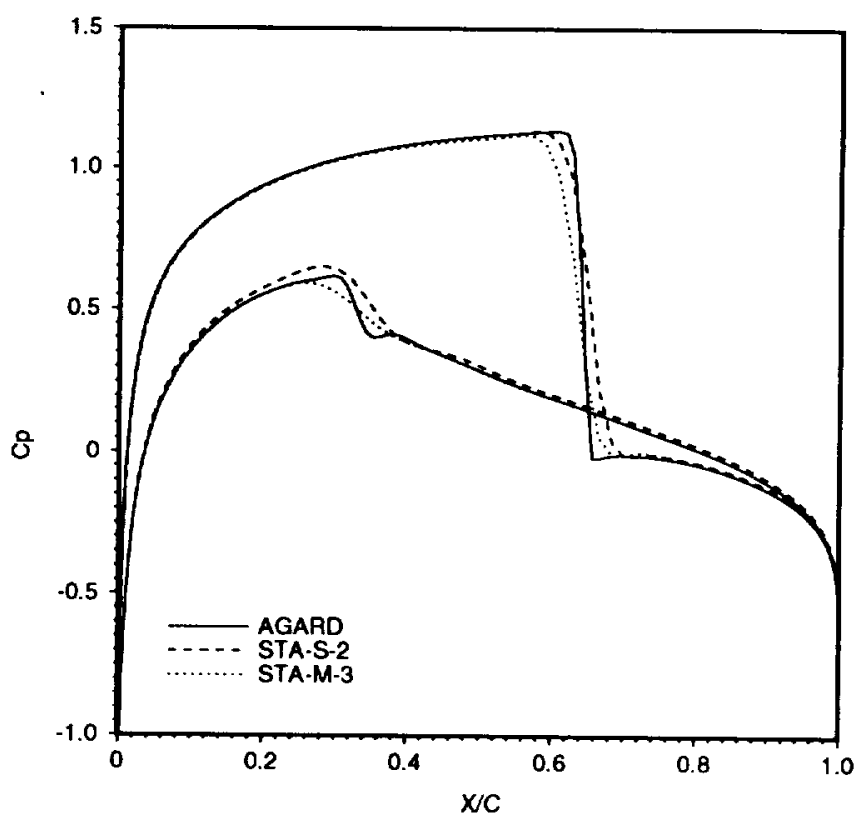

Figure 6. $C_{p}$ vs. $X / C$ plots for the finest AGARD solution [12], and the present coarse STA-S-2 and STA-M-3 solutions. 
UTOA-M-1

UTOA-S-1

$643 \times 131$

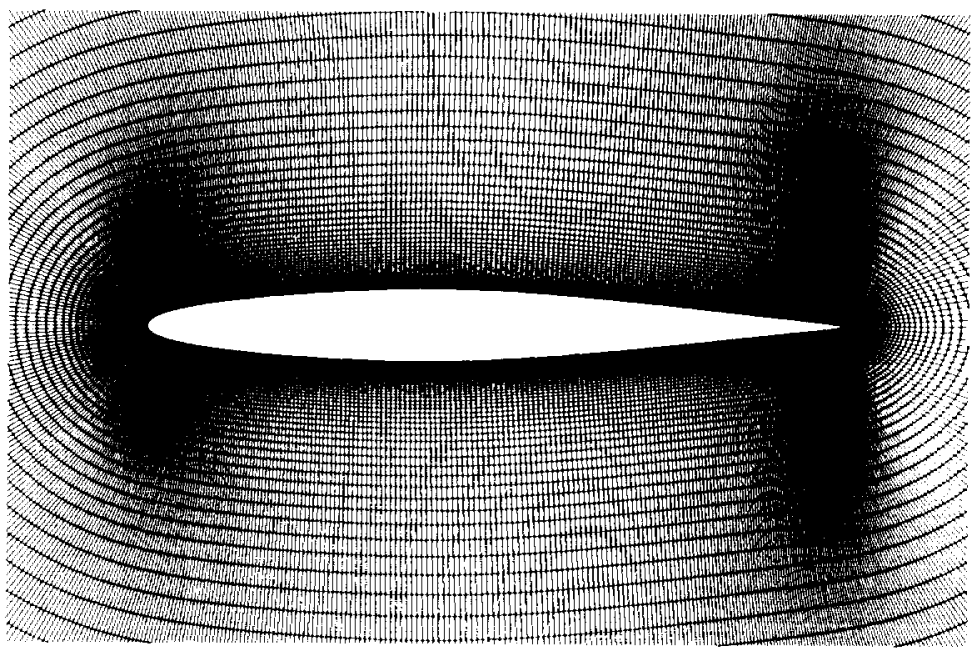

Figure 10. Decomposition of the UTOA-S-1 grid into 2, 16 , and 32 overlapping grid components. Intergrid boundary conditions are spatially conservative and have no interpolation error.

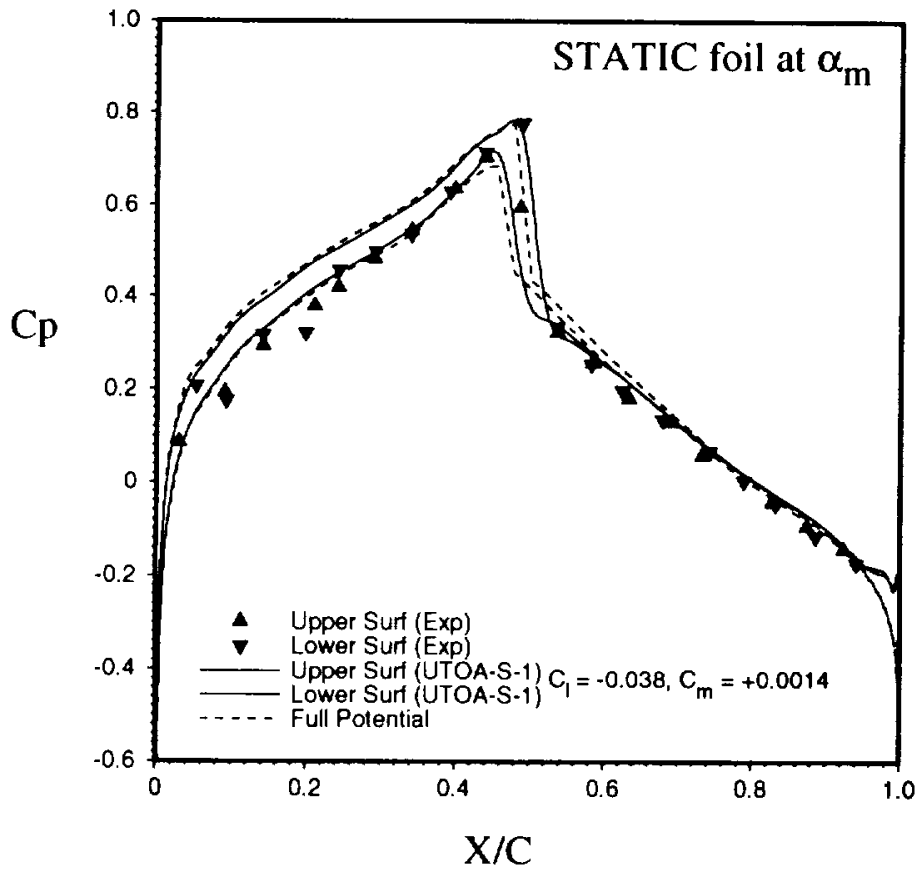

Figure 11. Cp vs $\mathrm{X} / \mathrm{C}$ comparison between NASA Experiment [13], the present UTOA-S-1 solution, and, for reference, a Full Potential solution $\left(M_{\infty}=0.796, \alpha_{m}=-0.21^{\circ}, \operatorname{Re}=1.256 \times 10^{7}\right)$.

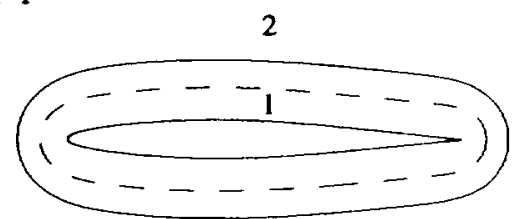

UTOA-M-2

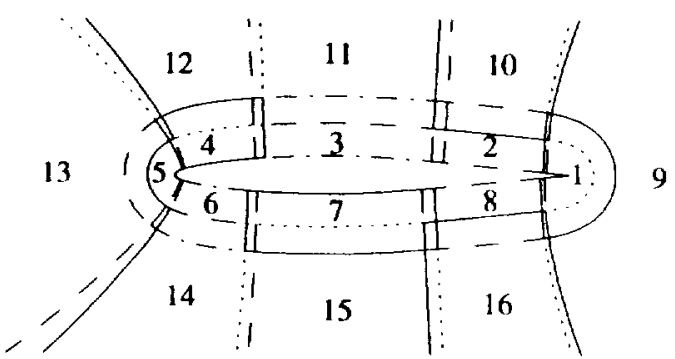

UTOA-M-3 (4 and 5)
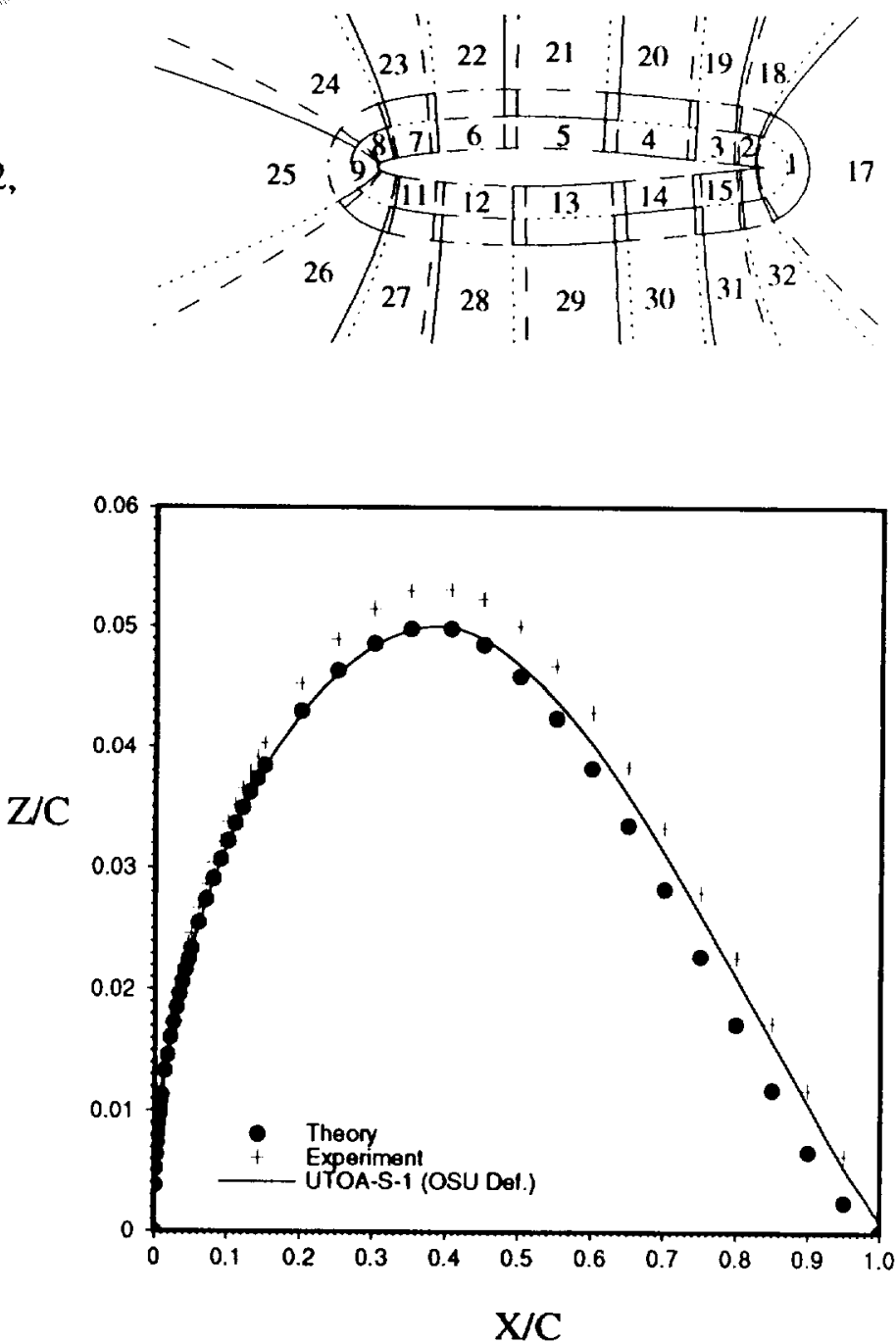

Figure 12. NACA 64A010 airfoil definitions.

a) Theoretical coordinates presented in [13],

b) coordinates of experimental configuration [13], and c) coordinates used in UTOA computations (based on OSU definition[14]). 
Table 1. Grid Refinement Cases

\begin{tabular}{|c|c|c|c|c|c|}
\hline Case $^{1}$ & $\operatorname{Grids}^{2}$ & Type & JM & $x$ & $\mathrm{KM}$ \\
\hline AGARD & 1 & $\mathrm{O}$ & 320 & $x$ & 64 \\
\hline STA-S-1 & 1 & 0 & 643 & $x$ & 131 \\
\hline STA-S-2 & 1 & 0 & 215 & $x$ & 44 \\
\hline$S T A-M-1$ & 2 & 0 & 643 & $x$ & 61 \\
\hline & & $\mathrm{BC}$ & 309 & $x$ & 195 \\
\hline \multirow[t]{2}{*}{ STA-M-2 } & 2 & 0 & 322 & $x$ & 31 \\
\hline & & $\mathrm{BC}$ & 155 & $x$ & 97 \\
\hline \multirow[t]{2}{*}{ STA-M-3 } & 2 & 0 & 215 & $x$ & 21 \\
\hline & & $\mathrm{BC}$ & 103 & $x$ & 65 \\
\hline \multirow[t]{2}{*}{ STA-M- 4} & 2 & 0 & 108 & $x$ & 11 \\
\hline & & $\mathrm{BC}$ & 51 & $\mathrm{x}$ & 33 \\
\hline \multirow[t]{7}{*}{ STA-AG } & 7 & O & 215 & $x$ & 21 \\
\hline & & $\mathrm{BC}$ & 103 & $x$ & 65 \\
\hline & & FBF & 25 & $\mathrm{x}$ & 21 \\
\hline & & $\mathrm{FBF}$ & 31 & $x$ & 21 \\
\hline & & FBF & 37 & $x$ & 17 \\
\hline & & $\mathrm{FBF}$ & 39 & $x$ & 21 \\
\hline & & $F C$ & 22 & $x$ & 25 \\
\hline \multicolumn{6}{|c|}{$\begin{array}{l}1 \text { Outer boundary is } 25 \text { chords } \\
\text { in all cases. } \\
2 \text { Type legend: } \\
O=\text { "O" topology } \\
\text { BC }=\text { Background Cartesian } \\
\text { FBF }=\text { Fine Body-Fitted } \\
\text { FC }=\text { Fine Cartesian }\end{array}$} \\
\hline
\end{tabular}

Table 2. Computed Loads

\begin{tabular}{|c|c|c|c|}
\hline Case & $\mathrm{C}_{\mathrm{I}}$ & $c_{1}$ & $C_{M}$ \\
\hline AGARD & 0.0230 & 0.3632 & -0.0397 \\
\hline scatter & \pm 0.0023 & \pm 0.0273 & \pm 0.0072 \\
\hline$S T A-S-1$ & 0.0145 & 0.3403 & -0.0359 \\
\hline STA-M-1 & 0.0155 & 0.3569 & -0.0394 \\
\hline STA $-M-2$ & 0.0148 & 0.3372 & -0.0357 \\
\hline STA-M-3 & 0.0152 & 0.3463 & -0.0361 \\
\hline STA $-M-4$ & 0.0195 & 0.3085 & -0.0319 \\
\hline$S T A-A G$ & 0.0162 & 0.3487 & -0.0358 \\
\hline \multicolumn{4}{|c|}{$\begin{array}{l}\text { * Scatter given in AGARD report [12] } \\
\text { is based on } 9 \text { Euler solutions to } \\
\text { the present flow conditions from } \\
\text { varying solvers, grid densities, } \\
\text { and grid type. }\end{array}$} \\
\hline
\end{tabular}

Table 3. Unsteady Transonic Oscillating Airfoil (UTOA) Test Conditions

\begin{tabular}{|c|c|c|}
\hline ID & 55 & NASA TM-81221* Case ID \\
\hline Foil & & NACA $64 A 010$ \\
\hline$M_{\infty}$ & 0.796 & Free Stream Mach \\
\hline$R_{e}$ & $12.56 \times 10^{6}$ & Reynolds Number \\
\hline$\alpha$ & $\boldsymbol{\alpha}_{\circ} \cos (\omega t)$ & $\begin{array}{c}\text { Oscillatory angle of } \\
\text { incidence }\end{array}$ \\
\hline $\boldsymbol{\alpha}_{\mathrm{m}}$ & $-0.21^{\circ}$ & Mean angle-of-attack \\
\hline $\boldsymbol{\alpha}_{\circ}$ & $1.01^{\circ}$ & Oscillatory pitch amplitude \\
\hline $\boldsymbol{x}$ & 0.202 & $\begin{array}{l}\text { Reduced frequency } \\
\boldsymbol{\kappa}=\omega \mathrm{l} / 2 \mathrm{U} \\
\omega=2 \pi \mathrm{f}\end{array}$ \\
\hline $\mathrm{f}$ & 34.4 & Frequency $(\mathrm{Hz})$ \\
\hline$x_{0} / C$ & 0.248 & Pitch axis $w / r$ leading edge \\
\hline 1 & 0.500 & Chord length (m) \\
\hline$C_{\infty}$ & 336 & Sonic speed $(\mathrm{m} / \mathrm{s})$ \\
\hline
\end{tabular}

Table 4. UTOA Test Cases

\begin{tabular}{|c|c|c|}
\hline Case & Grids & $\Delta t$ \\
\hline UTOA-S-1 & 1 & 0.0025 \\
\hline UTOA-M-1 & 2 & 0.0025 \\
\hline UTOA-M-2 & 16 & 0.0025 \\
\hline UTOA-M-3 & 32 & 0.0025 \\
\hline UTOA-M-4 & 32 & 0.0050 \\
\hline UTOA-M-5 & 32 & 0.0100 \\
\hline \multicolumn{3}{|c|}{$\begin{array}{l}\text { Case UTOA-S-1 is the } \\
\text { benchmark case. }\end{array}$} \\
\hline \multicolumn{3}{|c|}{$\begin{array}{l}\text { Cases UTOA-M-1 through } \\
\text { UTOA-M-5 are multiple } \\
\text { grids cases which have } \\
\text { zero interpolation error, } \\
\text { and are fully conserva- } \\
\text { tive. }\end{array}$} \\
\hline
\end{tabular}

